\title{
Metastatic thymic tumor arising from spermatic cord leiomyosarcoma
}

\author{
Kohei Aoki ${ }^{1}$ (D) Masatoshi Yamaguchi ${ }^{1} \cdot$ Ato Sugiyama $^{1} \cdot$ Yoshiaki Inoue $^{1} \cdot$ Hiroki Fukuda $^{1} \cdot$ Masatoshi Gika $^{1}$. \\ Mami Yamazaki $^{2} \cdot$ Ryutarou Kawano $^{2} \cdot$ Shuji Momose $^{2} \cdot$ Mitsuo Nakayama $^{1}$
}

Received: 30 March 2018 / Accepted: 17 July 2018 / Published online: 21 July 2018

(c) The Author(s) 2018

\begin{abstract}
We report the first known case of a metastatic thymic tumor arising from spermatic cord leiomyosarcoma, occurring in a 35 -year-old man. He had undergone an orchiectomy 24 months previously and a surgical excision of a subcutaneous metastasis 4 months prior to his current presentation. Computed tomography revealed a $1.5-\mathrm{cm}$, round-shaped anterior mediastinal mass. A thymectomy was performed and the diagnosis of metastatic leiomyosarcoma was made.
\end{abstract}

Keywords Thymus $\cdot$ Metastasis $\cdot$ Sarcoma

\section{Introduction}

The large majority of the neoplasms that arise in the thymus are of epithelial, lymphoid, or germ cell derivation [1]. Sarcomas of the thymus, whether the tumors are primary or metastatic, are extremely rare [2]. We describe herein a unique case of leiomyosarcoma presenting as a metastatic thymic tumor, which has not been previously reported.

\section{Case report}

An asymptomatic 35-year-old Japanese man was referred to us when an anterior mediastinal mass was found on computed tomography (CT) for a metastasis evaluation. He had had spermatic cord leiomyosarcoma 24 months previously. He had undergone a left radical orchiectomy and had received adjuvant radiation [3]. He had also undergone surgical excision of a subcutaneous metastasis 4 months prior to his current presentation. The mediastinal lesion had not been present on CT taken at that time. Physical examination

Kohei Aoki

aokikohe@saitama-med.ac.jp

1 Department of General Thoracic Surgery, Saitama Medical Center, Saitama Medical University, 1981 Kamoda, Kawagoe, Saitama 350-8550, Japan

2 Department of Pathology, Saitama Medical Center, Saitama Medical University, 1981 Kamoda, Kawagoe, Saitama 350-8550, Japan and laboratory findings were unremarkable. CT of the chest revealed a $1.5-\mathrm{cm}$, round-shaped, well-circumscribed anterior mediastinal mass (Fig. 1). No other lesion was identified. It was expected to be a malignant thymic tumor; therefore, total thymectomy was carried out by median sternotomy. The mass was located in the left lower part of the thymus, but the thymic capsule was intact. Grossly, within the left inferior lobe was a well-circumscribed and elastic tumor measuring $2.0 \times 1.4 \times 1.0 \mathrm{~cm}$. On cut section, the tumor appeared as a solid white tissue with slightly yellowish central necrosis (Fig. 2). On microscopic examination, it was composed of fascicles of atypical spindle cells with lightly eosinophilic cytoplasm, and large pleomorphic nuclei with numerous mitotic figures exceeding 10/10 highpower fields (Fig. 3). It was entirely surrounded on thymic tissues, and no lymph node tissue was identified around. Immunohistochemistry showed positive staining for desmin and $\alpha$-smooth muscle actin ( $\alpha$ SMA), and negative for S-100 protein, epithelial membrane antigen (EMA) and cytokeratin AE1/AE3, similar to the previously resected spermatic cord tumor, and negative for PAX8 and p63. Other areas of the thymic gland remained intact and seemed to be matched with age.

Based on the clinical presentation and surgical and histopathological findings, we concluded that this intra-thymic tumor was post-operative metastasis of spermatic cord leiomyosarcoma. After that he developed multiple metastases, subcutaneous, chest wall, adrenal gland, kidney, and pancreas and was resected. With progress of the disease, he died 55 months after the thymectomy. 


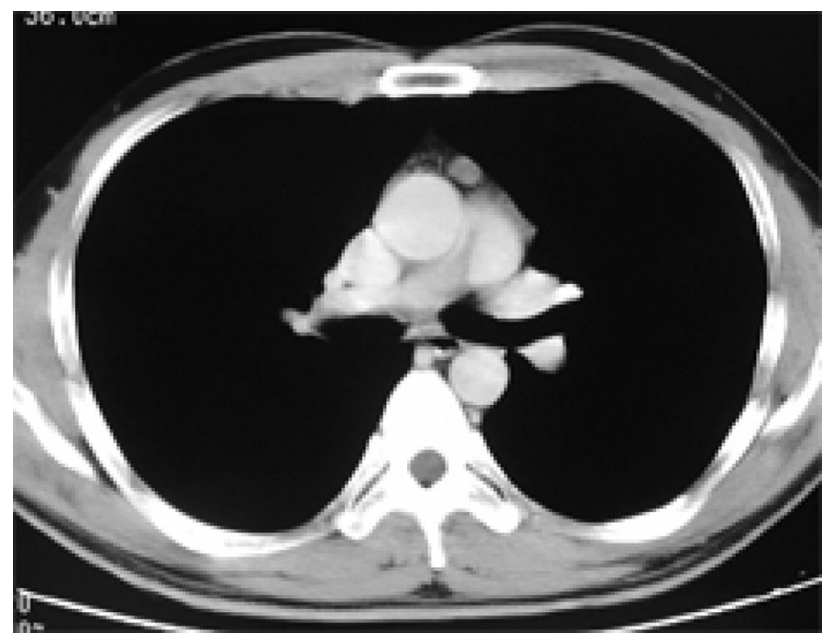

Fig. 1 Computed tomography of the chest revealing a $1.5-\mathrm{cm}$, roundshaped, well-circumscribed anterior mediastinal mass

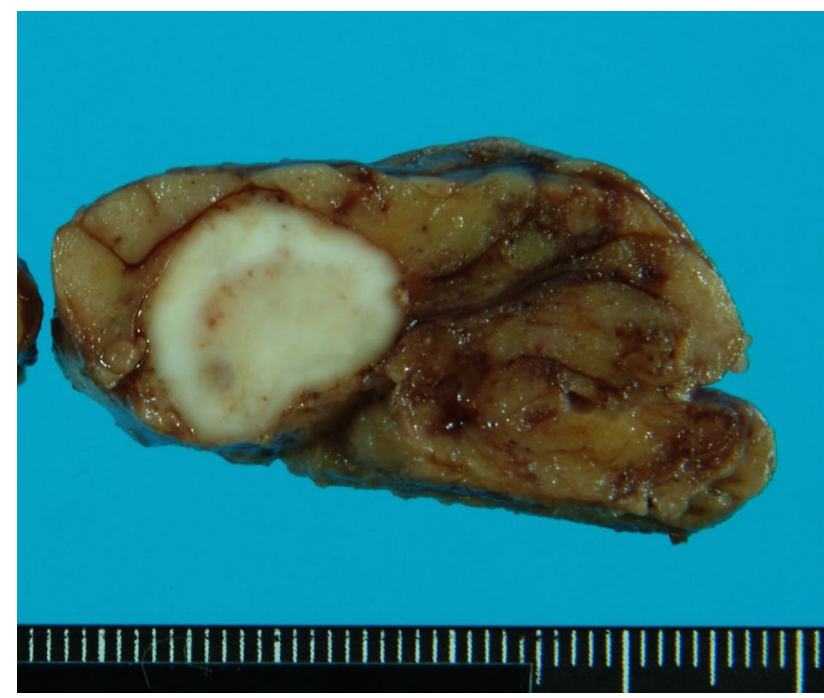

Fig. 2 Cut surface of the tumor showing a solid white tissue with slightly yellowish central necrosis

\section{Discussion}

The large majority of neoplasms that arise in the thymus are of epithelial, lymphoid, or germ cell derivation [1]. True sarcomas of the thymus are exceedingly rare and have not been well defined, although those of the anterior mediastinum have been reported [1, 4]. In the present case, the diagnosis of leiomyosarcoma was made by tumor morphology and immunohistochemical staining. The most important differential diagnosis is a sarcomatoid carcinoma of the thymus [5], but this possibility was excluded by the diffuse strong immunoreactivity for

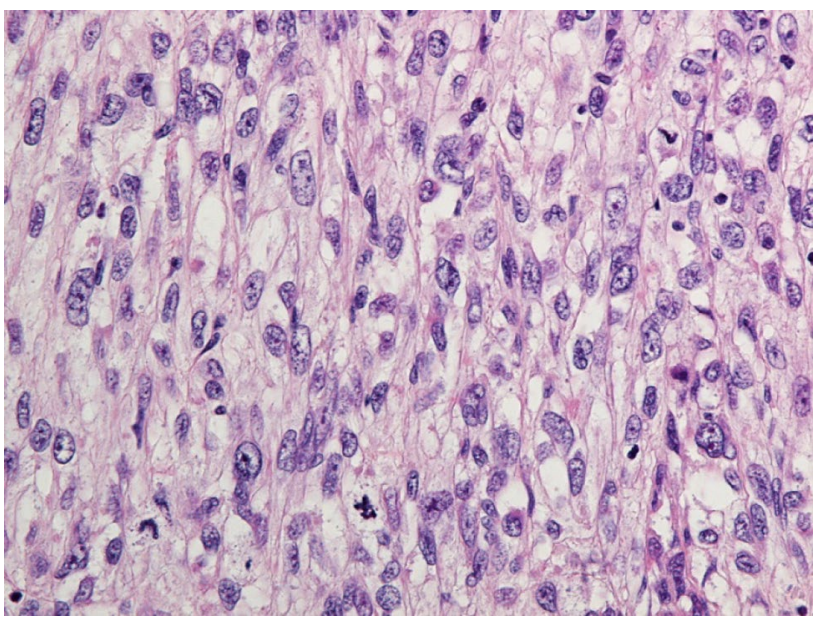

Fig. 3 Photomicrograph depicting fascicles of atypical spindle cells with lightly eosinophilic cytoplasm and large pleomorphic nuclei. (Hematoxylin and eosin; $\times 200$ original magnification)

desmin and $\alpha$ SMA, and the lack of cytokeratin or S-100 protein. Another differential diagnosis is a primary leiomyosarcoma arising in the thymus, but the present case had undergone resections of spermatic cord leiomyosarcoma and subcutaneous metastasis. Therefore, we concluded that this was most probably a thymic metastasis of spermatic cord leiomyosarcoma.

Metastatic carcinoma in the thymus is thought to be by no means uncommon. Middleton collected a series of autopsy thymuses and reported seven cases of thymic metastasis in 102 cases of carcinoma; the incidence was approximately $7 \%$ [6]. The most common primary tumors involving the thymus or anterior mediastinum are lung, thyroid, breast, colorectal and prostatic carcinomas, while melanoma and various sarcomas (liposarcoma, osteosarcoma, rhabdomyosarcoma, Kaposi sarcoma and malignant fibrous histiocytoma) are rare primary tumors [2]. However, to the best of our knowledge, metastatic thymic leiomyosarcoma has not been previously reported.

While sarcomas of any organs, including spermatic cord leiomyosarcoma, often metastasize hematogenously to chest cavity structures, the result is usually lesions of the pulmonary parenchyma $[7,8]$. The thymus or anterior mediastinum is regarded as an extremely unusual site for metastasis from distant primary sarcomas, particularly in the absence of significant pulmonary parenchymal disease. The present case demonstrates an extremely atypical presentation of metastatic leiomyosarcoma in the absence of any overt pulmonary parenchymal disease or other metastatic lesions with the exception of spread to the subcutaneous tissue at the right shoulder 4 months prior to thymic tumor discovery.

In regard to surgical indications for metastatic thymic tumors, especially sarcomas that are often ineffective in 
chemotherapy and radiotherapy, it is considered reasonable to resect if possible to remove completely. However, we should consider less invasive procedure such as thoracoscopic surgery.

In summary, for patients who are found to have a solitary tumor in the thymus, while the standard differential diagnosis of primary tumors of the thymus deserves the highest consideration, metastatic thymic tumor may merit consideration as well.

\section{Compliance with ethical standards}

Conflict of interest The authors have declared that no conflict of interest exists.

Open Access This article is distributed under the terms of the Creative Commons Attribution 4.0 International License (http://creativeco mmons.org/licenses/by/4.0/), which permits unrestricted use, distribution, and reproduction in any medium, provided you give appropriate credit to the original author(s) and the source, provide a link to the Creative Commons license, and indicate if changes were made.

\section{References}

1. Shimosato Y, Mukai K. Tumors of the mediastinum. In: Atlas of tumor pathology, third series, fascicle 21. Washington, D.C.: Armed Forces Institute of Pathology; 1997. pp. 227-8.
2. Muller-Hermelink HK, Strobel Ph, Zettl A, Marx A. Metastases to thymus and anterior mediastinum. In: Tarvis WD, Brambilla E, Muller-Hermelink HK, Harris CC, editors. Pathology and genetics of tumours of the lung, pleura, thymus and heart. Lyon: World Health Organization Classification of Tumours, IARC Press; 2004. pp. 247-8.

3. Ishii N, Yoshida S, Yoshinaga A, Ohno R, Terao T, Watanabe T, et al. Primary leiomyosarcoma of the spermatic cord with an unusually indolent clinical course. Hinyokika Kiyo. 2006;52:159-61.

4. Swanson PE. Soft tissue neoplasms of the mediastinum. Semin Diagn Pathol. 1991;8:14-34.

5. Rosai J. Histological typing of tumours of the thymus. World Health Organization International histological classification of tumours. 2nd ed. Berlin: Springer; 1999.

6. Middleton G. Involvement of the thymus by metastatic neoplasms. Br J Cancer. 1966;20:41-6.

7. Potter DA, Glenn J, Kinsella T, Glatstein E, Lack EE, Restrepo C, et al. Patterns of recurrence in patients with high grade soft tissue sarcoma. J Clin Oncol. 1985;3:353-66.

8. Ballo MT, Zagars GK, Pisters PW, Feig BW, Patel SR, von Eschenbach AC. Spermatic cord sarcoma: outcome, patterns of failure and management. J Urol. 2001;166:1306-10. 